\title{
Black Body Radiation for Students and Secondary Schools
}

\author{
http://dx.doi.org/10.3991/ijoe.v10i5.3750 \\ Andreas Pester, Christian Kreiter, Ramona Oros \\ Carinthia University of Applied Sciences, Villach, Austria
}

\begin{abstract}
Online laboratories in the field of science are gaining a lot of interest in optic field because a great number of simulations can be found but almost no remote labs. Black body radiation is a hard topic specially for secondary school students so having the possibility to really see how the phenomena happen will facilitate their learning process.
\end{abstract}

Index Terms_remote lab, online course, black body.

\section{INTRODUCTION}

The following work describes an online course in a field of optics that has the purpose to help students understand the black body concept better. Course structure consists of theoretical material, an interactive remote experiment and exercises. The theoretical part of the course describes the concept of a black body and its radiation, as well as the existence of the phenomenon in real life and in nature. Also some other physical parameters that would help get deeper in the physical phenomena the experimental part of the course provides the details of the online lab and the remote experiment, which allows students to relate the experiment with the natural process of daily life.

\section{BLACK BODY RADIATION LAB}

The first experiment presents the history of the measurement and shows how the radiation power of a light source behaves over time. Thus it can be seen that at the beginning, when the light source is cooler and the electric current is passing through the filament, the resistance of the bulb is lower. As the light bulb warms up, his its resistance increases with the temperature, but the current through the light bulb remains constant. Related to this is the power distribution over distance. This power can be measured with different sensors since the light represents a form of electromagnetic radiation; it decreases in intensity the further it travels from the emission point. Theoretical concept of Inverse square law is used to predict the rate of illumination of a light source.

To realize a system that respond to all needs of the experiments it has specific hardware and software elements.

\section{A. Hardware}

The equipment of the black body laboratory can be seen in Figure 1 and contains the main following components:

- A plate with four different light sources and one heater element - energy saver bulb (11W), LED (8.1 $\mathrm{W} ; 4001 \mathrm{~m})$, halogen lamp $(20 \mathrm{~W})$, regular light bulb $(60 \mathrm{~W})$ and a heat element

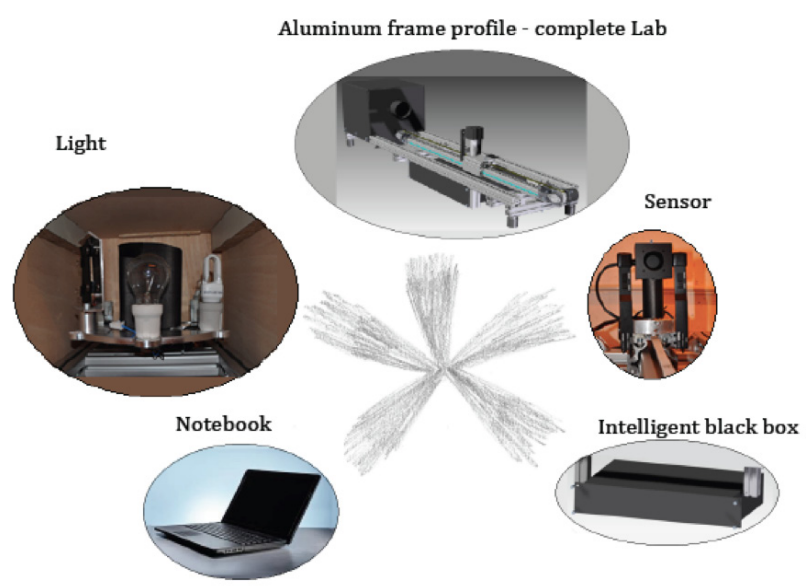

Figure 1. Hardware components of blackbody system

- Three sensors - two photodiode sensors of $\lambda=700 \mathrm{~nm}$ $-1800 \mathrm{~nm}$ and $\lambda=200 \mathrm{~nm}-1100 \mathrm{~nm}$ and one thermal sensor of $\lambda=190 \mathrm{~nm}-25 \mu \mathrm{m}$

- An intelligent black box - control boards and data acquisition cards

\section{B. Software}

The software used to control all mechanical and electrical components from the black body radiation laboratory system is LabVIEW. Therefore the Blackbody Radiation.vi was created to develop experiments based on the physical concept and keep the user interface structure as close as possible as the hands-on lab looks like.

The black body radiation laboratory application consists of two experiments. Users are able to measure the power distribution of the radiation sources over time and over distance (Figure 2).

The structure of the interface is commune for both experiments. Input parameters are situated in the right side and from there users can choose the light source that they want to measure, the sensor, the distance between the light source and the sensors and the power of the light source on the left side the output data of the experiments will be presented. This information appears in a graphical way and also offers the possibility to export it in a text file for further analyses.

During performance of the experiment no input can be changed. 


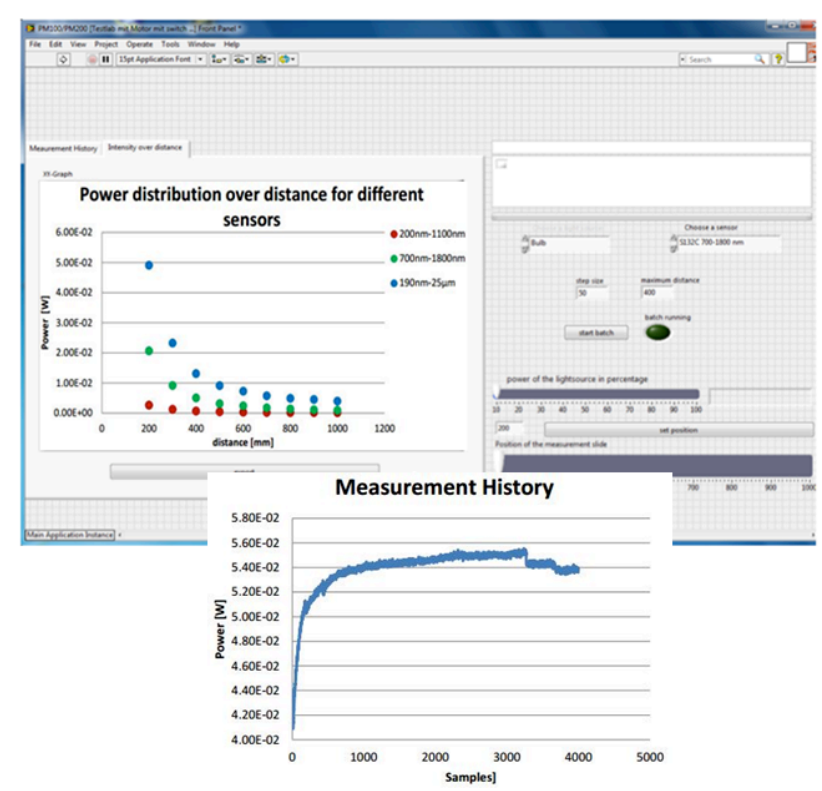

Figure 2. User interface Blackbody Radiation experiments

\section{IMPLEMENTATION PLAN}

The Blackbody Radiation lab has been included into the secondary schools program in some schools from Austria and other countries that were partner in the OLAREX project (Figure 3), framework in which this lab was developed.
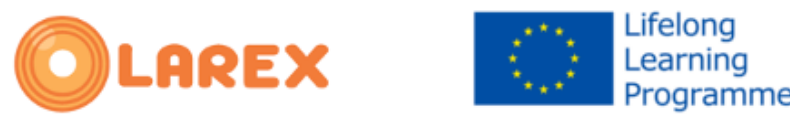

Figure 3. OLAREX

The online course structure was realized in collaboration with school teachers to fulfill their need and to be close to each countries curriculum. Students can apply their theoretical knowledge from their courses by using this remote lab and then analyses can be organized in groups of students to discuss their results.

\section{CONCLUSIONS}

This laboratory enables remote users to observe and learn the physical process that occurs in the laboratory without the necessity to get in contact with the lab equipment of the blackbody system.

The main advantage of such a system represents the fact that users, without having real equipment, can access and perform real experiments over internet without the need to buy them.

\section{OUTLOOK}

Since the current experiment setup already acts like a batched lab - in short: a lab where the user settings are put on a batch and executed one after another - the biggest constraint is the user interface, which allows only one user at a time to work with the lab. Furthermore the installation of the LabVIEW Runtime Engine is needed to access the lab via browser. With further development the black body radiation lab will get a new interface, which allows multiple users to access the lab and relies solely on HTML and JavaScript on the client side. Thus, experimentation with the blackbody radiation lab will be simplified for every user and even tablet users will have then the possibility to access the lab.

\section{ACKNOWLEDGMENT}

This work has been carried out within the project "Open Learning Approach with Remote Experiments (OLAREX)". The project is supported by the Lifelong Learning Programme of the European Union within the transversal programme, Key Activity 3 - ICT (project No. 518987-LLP-1-2011-1-ES-KA3-KA3MP). The opinions expressed by the authors do not necessarily reflect the position of the European Community, nor does it involve any responsibility on its part.

\section{REFERENCES}

[1] G. Z. Danilo, P. Andreas, A. Michael, M. Christian, " Remote Applications and Trends", E-pragmatic module, 2011,

[2] G. Z. Danilo, A. Michael, "Work in Progress Integrating Educational Online Lab Platforms around the iLab Shared Architecture", 41st ASEE/IEEE Frontiers in Education Conference, Rapid City, 2011

[3] Zutin, D. G. (2009). A Network of Online Labs based on the iLab Shared Architecture (ISA). (Master's Thesis). Carinthia University of Applied Sciences. Villach, Austria

[4] Niederstätter, M. (2010). Lab2go - A Repository to Locate Online Laboratories. (Master's Thesis). Carinthia University of Applied Sciences. Villach, Austria

[5] P. Andreas, O. Ramona, D. Olga, "Explorative Learning with technology in STEM - the OLAREX experience", ICL 2013, Kazan, Russia, 25 - 27 September 2013

[6] P. Andreas, O. Ramona, D. Olga, "OLAREX : Initiating Secondary Schools Teachers into Online Labs Experience for Teaching ", ECEL 2013, Sophia Antipolis, France, 30 - 31 October 2013

[7] P. Andreas, O. Ramona, "iLab based remote labs as an approach for collaborative learning in STEM subjects", ICBL 2013, Florianopolis, Brazil, 6 - 8 November 2013

\section{AUTHORS}

Andreas Pester, Christian Kreiter, and Ramona Oros are with System Engineering and IT - Carinthia University of Applied Sciences, Villach, Austria.

Submitted 07 April 2014. Published as resubmitted by the authors 13 September 2014. 